\title{
EDITORIAL
}

\section{The role of interventional radiology in the management of hemodynamically compromised patients}

\author{
Mohammad Arabi ${ }^{*}\left(\mathbb{D}\right.$, Joseph J. Gemmete ${ }^{2}$ and Yaseen Arabi ${ }^{3}$
}

๑ 2018 Springer-Verlag GmbH Germany, part of Springer Nature and ESICM

\section{Introduction}

The minimally invasive nature of interventional radiology (IR), supported by the advances in imaging and improvements in endovascular techniques, has resulted in expansion of its role as an alternative option for medical, endoscopic, or surgical interventions. While IR procedures are currently incorporated in several medical, oncological, and surgical practice guidelines, the levels of recommendations mainly depend on low-quality evidence lacking long-term outcomes and comparison to other treatment options. In this editorial, we provide an overview of the emerging role of IR procedures in management of hemodynamically compromised patients based on current clinical practice guidelines, with a focus on life-threatening bleeding, massive and submassive pulmonary embolism (PE), and sepsis. A summary of the related clinical practice guidelines is presented in Table 1. Angiographic images of selected representative cases are shown in Online Supplement Fig. 1.

\section{Life-threatening bleeding}

IR procedures have an important role in several conditions related to non-traumatic and traumatic bleeding (Table 1) (Supplement Fig. 1, panels 1 and 2). Although practice guidelines addressed the role of IR procedures in specific types of injuries and pathologies, the same concept of embolization and bleeding control can be generalized to other body parts and types of traumatic injuries.

\section{Non-variceal gastrointestinal (GI) bleeding}

Transarterial embolization using different embolic agents can be considered as an alternative to surgery in patients with GI bleeding after failed medical or endoscopic management. Transarterial embolization achieves control of the bleeding site in $40-80 \%$ of cases depending on the site of hemorrhage $[1,2]$. The source of non-variceal bleeding is often identified by upper or lower endoscopy. In patients with an unidentified source with significant active bleeding (estimated at $0.5 \mathrm{~mL} / \mathrm{min}$ or more), angiography can be used to identify the source followed by embolization. In patients with less active bleeding, $\mathrm{CT}$ angiography typically detects bleeding that exceeds $0.3-0.5 \mathrm{~mL} / \mathrm{min}$, and scintigraphy with ${ }^{99 \mathrm{~m}} \mathrm{Tc}$-labeled red blood cells can help identify the source of slower bleeds $(0.05-0.1 \mathrm{~mL} / \mathrm{min})$. Once active extravasation is diagnosed, embolization of the bleeding vessel can follow. Although patients who are treated by transarterial embolization tend to be older and have more medical comorbidities than those managed with surgery, transarterial embolization is associated with lower morbidity and similar survival rates compared to surgery $[1,2]$. However, 


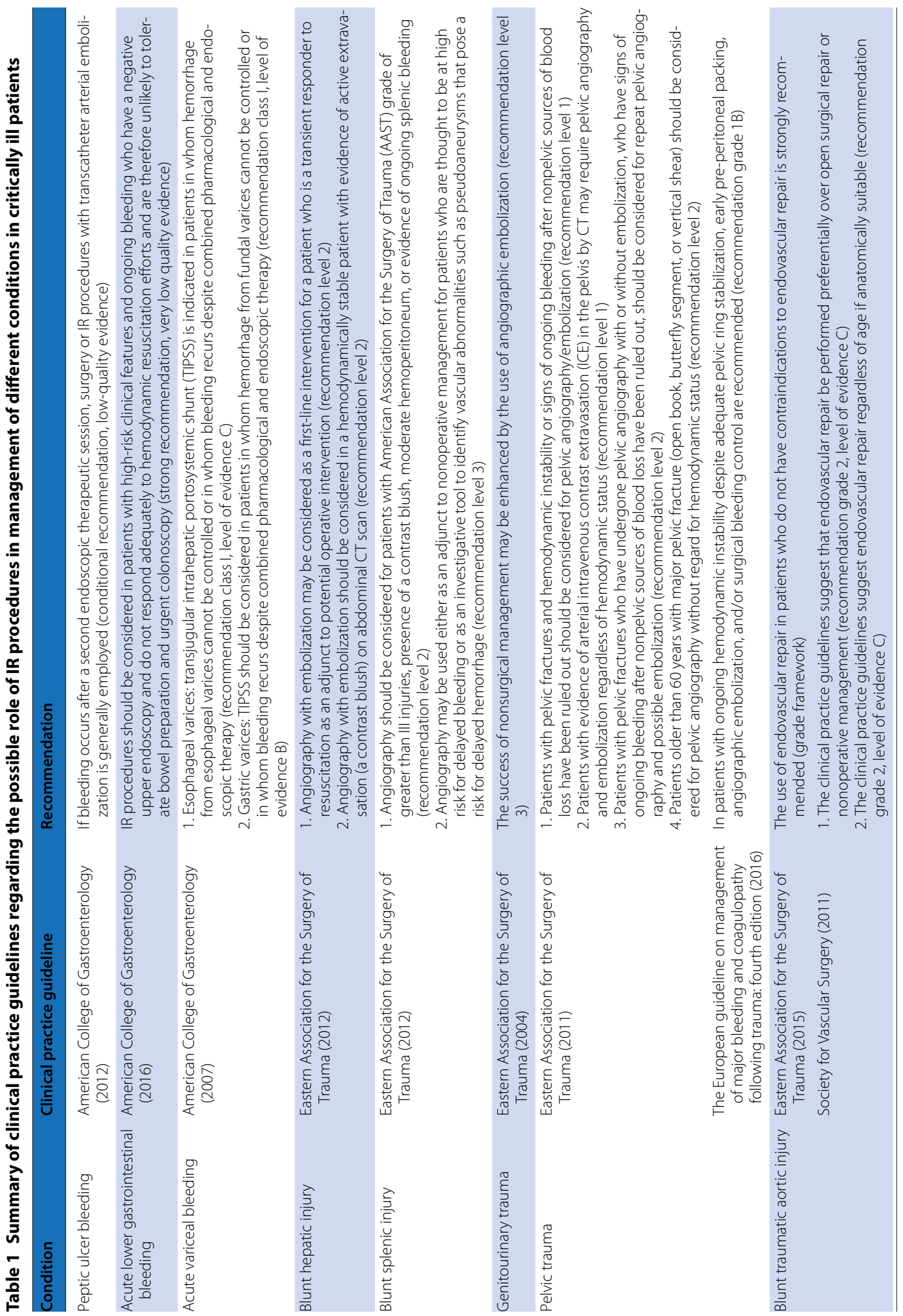




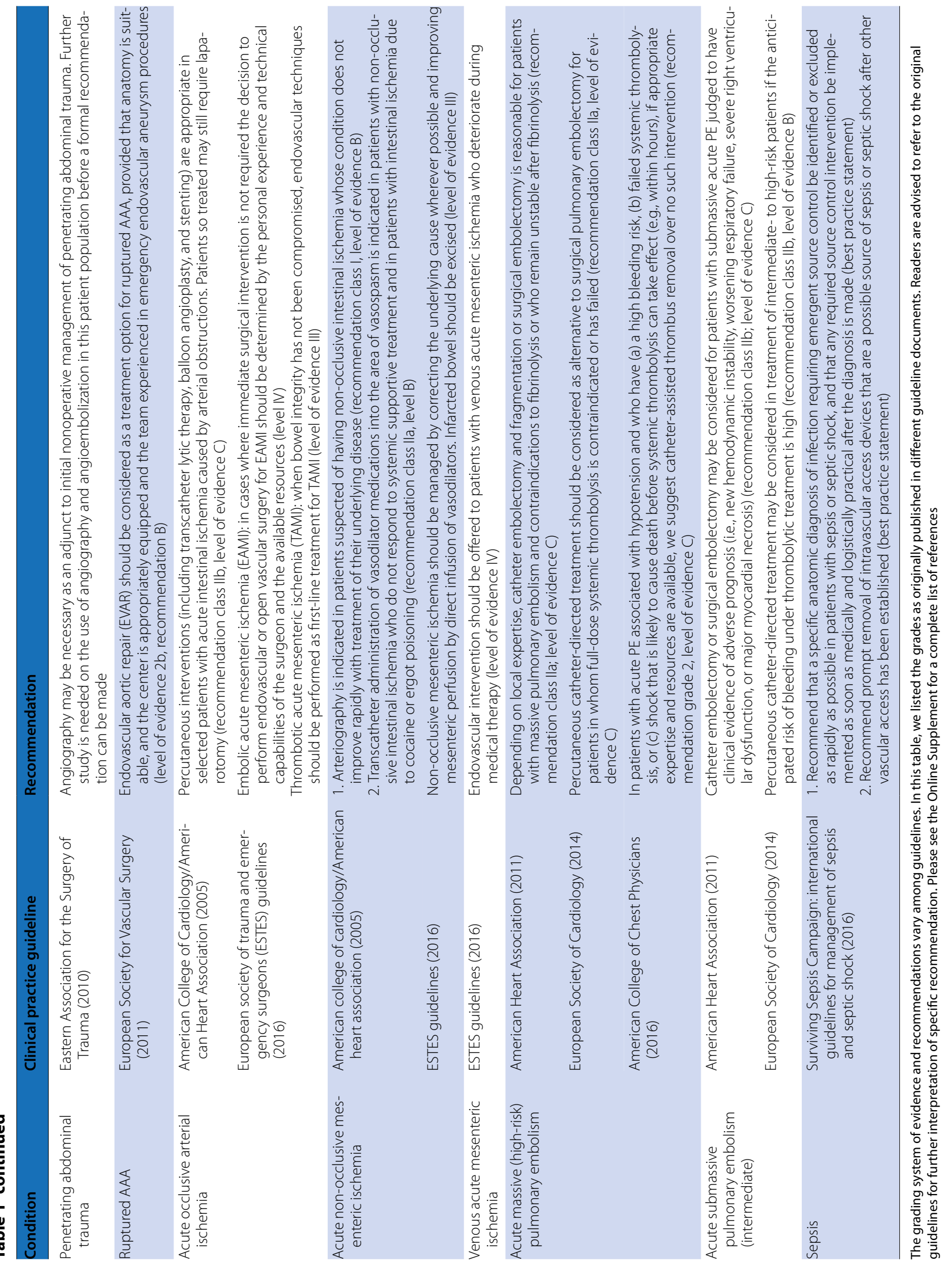


re-bleeding rate is higher with transarterial embolization compared to surgery and it is often related to coagulation disorders, inaccurate angiographic localization of bleeding, or inadequate embolization.

\section{Variceal bleeding}

In patients with acute esophageal variceal bleeding, the first line of therapy is endoscopic gastroesophageal variceal ligation or sclerotherapy combined with pharmacologic therapy [3]. Transjugular intrahepatic portosystemic shunt (TIPSS) is indicated in patients in whom hemorrhage from esophageal varices cannot be controlled or in whom bleeding recurs despite combined pharmacological and endoscopic therapy [3]. Results from randomized clinical trials suggest that TIPSS may be more effective than endoscopic and medical management in reducing the rate of recurrent variceal bleeding, without a survival benefit $[4,5]$. Early creation of TIPSS within $72 \mathrm{~h}$ after admission is associated with lower rates of treatment failure and mortality compared to a combination of vasoactive therapy, beta-blockers, endoscopic ligation, and rescue TIPSS [6]. TIPSS is associated with higher rates of early hepatic encephalopathy $[4,5]$. Additional IR procedures for the management of variceal bleeding include balloon-occluded retrograde transvenous obliteration (BRTO) of gastric varices, transhepatic embolization of gastroesophageal varices, portal vein recanalization/thrombectomy with or without TIPSS, and partial splenic artery embolization.

\section{Solid organ injury}

The Eastern Association for the Surgery of Trauma (EAST) guidelines recommend surgical interventions for the management of solid organ abdominal traumatic injuries in hemodynamically unstable patients. The same guidelines suggest embolization as an adjunctive treatment in hemodynamically stable patients responding to resuscitative measures with evidence of active extravasation, or in those at high risk for the development of delayed bleeding from vascular injuries or pseudoaneurysms [7]. Published series report clinical success rates of hepatic, splenic, and renal embolization of $80 \%, 85 \%$, and $90 \%$, respectively [8], although recurrent bleeding occurs in up to 25\% depending on the American Association for the Surgery of Trauma (AAST) grade of injury and embolization techniques [8].

\section{Blunt traumatic aortic injuries}

Endovascular repair with stent grafting is increasingly being performed in lieu of open surgical repair owing to improvement of stent design and delivery (Supplement Fig. 1, panel 2). Findings from the RESCUE prospective nonrandomized multicenter study and the AAST multicenter study demonstrate that endovascular aortic repair, compared to open surgical repair, is associated with significantly less blood loss, lower incidence of stroke, paraplegia, and mortality, and shorter hospital stay [9].

\section{Massive and submassive PE}

Based on current evidence, the 2016 American College of Chest Physicians guidelines suggest the use of systemic thrombolysis over catheter-directed therapy (CDT) in patients with acute PE [10]. Nonetheless, if appropriate expertise and resources are available, the use of CDT is suggested in patients with acute PE who have hypotension and are at a high risk of bleeding or have failed systemic thrombolysis or are in shock that is likely to cause death before systemic thrombolysis can take effect [10]. CDT can be mechanical and/or pharmacological. There are several low-profile catheters (6-10 Fr) used to facilitate pulmonary reperfusion. The use of a specific catheter depends on operator's experience, risk for bleeding, patient's condition, and the need for immediate reperfusion. Mechanical CDT entails either thrombus disruption or fragmentation. Alternatively, thrombus removal can be performed using manual suction catheters or thrombectomy devices. Pharmacological CDT uses specific infusion catheters, with or without ultrasound agitation, allowing for administration of lower doses of thrombolytics compared to systemic therapy and mechanical device for thrombus removal to further accelerate pulmonary reperfusion (Supplement Fig. 1, panel 3). Given the emerging nature of some of these techniques, data about the effectiveness of each device are derived mainly from observational studies. A recent systematic review reported 16 prospective and retrospective studies between 2008 and 2016 and included 860 patients primarily treated with ultrasound-assisted CDT with a mean tPA dose of $24 \mathrm{mg}$ [11]. This analysis showed that ultrasound-assisted CDT was associated with a significant decrease in pulmonary artery systolic pressures (mean drop of $15 \mathrm{mmHg}$ ) and significantly reduced the right ventricle/left ventricle ratio [11]. The rate of major bleeding was estimated at $4.7 \%$, most requiring transfusion only, while intracranial hemorrhage occurred in $0.35 \%$ [11]. The in-hospital mortality of massive PE following CDT was $13 \%$ and $0.7 \%$ for submassive PE [11]. Given the complexity and the multidisciplinary nature of management of massive and submassive PE, the concept of pulmonary embolism response team (PERT) has emerged, in which intensivists and interventional radiologists are typically core members [12]. Initial data describing the experience with PERT showed that up to $18 \%$ of patients received CDT, $5-11 \%$ of patients were 
given systemic thrombolytics, and nearly $70 \%$ of patients received anticoagulation only $[13,14]$.

\section{Sepsis}

The Surviving Sepsis Campaign guidelines for management of sepsis and septic shock recommend early identification and control of specific anatomic source of infection using the least invasive effective option to avoid complications in these critically ill patients [15]. IR procedures are minimally invasive and often effective in source control of infection sites that are amenable for drainage, such as percutaneous drainage of abscesses, percutaneous transhepatic biliary drainage, and cholecystostomy and nephrostomy tube placement [15]. Surgical intervention should be considered when other IR procedures are inadequate or cannot be provided in a timely fashion [15].

In conclusion, IR procedures have an important role in properly managing selected hemodynamically compromised critically ill patients. While IR procedures carry generally lower morbidity, further studies are needed to evaluate survival and long-term outcomes in comparison to medical or surgical alternatives.

\section{Electronic supplementary materia}

The online version of this article (https://doi.org/10.1007/s00134-018-5236-3) contains supplementary material, which is available to authorized users.

\section{Author details}

${ }^{1}$ Department of Medical Imaging, Division of Vascular Interventional Radiology, King Abdulaziz Medical City, National Guard Health Affairs, P.O. Box 22490, Mail code 1222, Riyadh 11426, Saudi Arabia. ${ }^{2}$ Departments of Radiology and Neurosurgery, University of Michigan Health Centers, Ann Arbor, MI, USA. ${ }^{3}$ Intensive Care Department, King Abdulaziz Medical City, Riyadh, Saudi Arabia.

\section{Compliance with ethical standards}

\section{Conflicts of interest}

On behalf of all authors, the corresponding author states that there is no conflict of interest.

Received: 12 April 2018 Accepted: 18 May 2018

Published online: 13 June 2018

\section{References}

1. Beggs AD, Dilworth MP, Powell SL, Atherton H, Griffiths EA (2014) A systematic review of transarterial embolization versus emergency surgery in treatment of major nonvariceal upper gastrointestinal bleeding. Clin Exp Gastroenterol 7:93-104

2. Kyaw M, Tse Y, Ang D, Ang TL, Lau J (2014) Embolization versus surgery for peptic ulcer bleeding after failed endoscopic hemostasis: a meta-analysis. Endosc Int Open 2:E6-E14

3. Garcia-Tsao G, Sanyal AJ, Grace ND, Carey W, Practice Guidelines Committee of the American Association for the Study of Liver Diseases; Practice Parameters Committee of the American College of Gastroenterology (2007) Prevention and management of gastroesophageal varices and variceal hemorrhage in cirrhosis. Hepatology 46:922-938

4. Holster IL, Tjwa ET, Moelker A et al (2016) Covered transjugular intrahepatic portosystemic shunt versus endoscopic therapy + beta-blocker for prevention of variceal rebleeding. Hepatology 63:581-589

5. Rossle M, Deibert P, Haag K et al (1997) Randomised trial of transjugularintrahepatic-portosystemic shunt versus endoscopy plus propranolol for prevention of variceal rebleeding. Lancet 349:1043-1049

6. Garcia-Pagan JC, Caca K, Bureau C et al (2010) Early use of TIPS in patients with cirrhosis and variceal bleeding. N Engl J Med 362:2370-2379

7. Stassen NA, Bhullar I, Cheng JD et al (2012) Nonoperative management of blunt hepatic injury: an Eastern Association for the Surgery of Trauma practice management guideline. J Trauma Acute Care Surg 73:S288-S293

8. Gould JE, Vedantham S (2006) The role of interventional radiology in trauma. Semin Intervent Radiol 23:270-278

9. Khoynezhad A, Azizzadeh A, Donayre CE et al (2013) Results of a multicenter, prospective trial of thoracic endovascular aortic repair for blunt thoracic aortic injury (RESCUE trial). J Vasc Surg 57(899-905):e891

10. Kearon C, Akl EA, Ornelas J et al (2016) Antithrombotic therapy for VTE disease: chest quideline and expert panel report. Chest 149:315-352

11. Bloomer TL, El-Hayek GE, McDaniel MC et al (2017) Safety of catheterdirected thrombolysis for massive and submassive pulmonary embolism: results of a multicenter registry and meta-analysis. Catheter Cardiovasc Interv 89:754-760

12. Barnes GD, Kabrhel C, Courtney DM et al (2016) Diversity in the pulmonary embolism response team model: an organizational survey of the National PERT Consortium members. Chest 150:1414-1417

13. Carroll BJ, Pemberton H, Bauer KA et al (2017) Initiation of a multidisciplinary, rapid response team to massive and submassive pulmonary embolism. Am J Cardiol 120:1393-1398

14. Kabrhel C, Rosovsky R, Channick R et al (2016) A multidisciplinary pulmonary embolism response team: initial 30-month experience with a novel approach to delivery of care to patients with submassive and massive pulmonary embolism. Chest 150:384-393

15. Rhodes A, Evans LE, Alhazzani W et al (2017) Surviving Sepsis Campaign: international guidelines for management of sepsis and septic shock: 2016. Intensive Care Med 43:304-377 